\title{
Numerical Simulations of KPG 302 (NGC 3893/96)
}

\author{
Margarita Rosado, ${ }^{1}$ Ruslan Gabbasov ${ }^{2}$ and Isaura Fuentes-Carrera ${ }^{3}$ \\ ${ }^{1}$ Instituto de Astronomía \\ Universidad Nacional Autónoma de México \\ Apartado Postal 70-264, México, D.F., CP 04510 \\ email: margarit@astroscu.unam.mx \\ ${ }^{2}$ Instituto de Astronomía \\ Universidad Nacional Autónoma de México \\ Apartado Postal 70-264, México, D.F., CP 04510 \\ email: gabbasov@astro. unam.mx \\ ${ }^{3}$ Escuela Superior de Física y Matemáticas, Instituto Politécnico Nacional (ESFM-IPN) \\ Edificio 9, U.P. Adolfo Lopez Mateos, México, D.F., CP07730 \\ email: isaura.fuentescarrera@gmail.com
}

\begin{abstract}
We present the results of numerical simulations on the M51-type galaxy pair KPG 302 (NGC 3893/96) taking as boundary conditions for these simulations the results of our observations on the kinematics of this pair. The observations allow us to deduce the different components of each galaxy in the pair (stellar disk and bulge, dark matter halo) as well as the geometry of the encounter at the present time. The numerical simulations were carried out using GADGET2 numerical code including gas. We describe in detail how the numerical simulations are being done. We succeeded to reproduce the actual conditions of the pair, in particular, the granddesign spiral arms of NGC 3893 (the main galaxy of the pair) when the numerical simulations include gas, consider a parabolic direct collision and multiple passages.
\end{abstract}

Keywords. galaxies: interactions, galaxies: kinematics and dynamics, (cosmology:) dark matter

\section{Introduction}

The simplest case in the process of galaxy interactions are the Isolated Galaxy Pairs (IGPs). IGPs are systems composed of two galaxies located in the space so closely that the gravitational effect of their nearest neighbors can be neglected relative to the gravitational effects exerted between each other. We are conducting a comprehensive study of IPGs starting by carring out observations on their kinematics by means of the PUMA FabryPerot instrument. In this way, we have obtained the kinematics of about $15 \%$ of the IGPs of the Catalogue of Karachentsev (1972). Among the IPGs there are the interacting galaxy pairs of the M51-type are pairs formed of a large spiral galaxy (host) and a less massive companion (satellite), with some sign of interaction. These pairs are relevant in many ways but here we focus on the fact that for these pairs there are at least two independent ways of estimating the dark matter content of the large galaxy: (1) by means of the rotation curve (RC) of the host, and (2) by means of the orbital motion of the satellite galaxy around the host galaxy. NGC 3893/96 is an interacting galaxy pair with number 302 in the Karachentsev Catalogue, KPG (Karachentsev 1972). NGC 3893 is a grand-design Sc type galaxy similar to M51. Its companion, NGC 3896, appears to be a lenticular galaxy (S0a). Radio images show extended HI emission encompassing both galaxies (Verheijen \& Sancisi 2001). This common HI envelope is elongated from SE to NW, parallel to the line that joins the nuclei of both galaxies. The kinematical 
analysis derived from the PUMA Fabry-Perot observations has been already published in Fuentes-Carrera et al. (2007).

\section{Observations and main kinematical results}

Observations of NGC 3893/96 (KPG 302) were done at the $2.1 \mathrm{~m}$ telescope at the OANSPM (México) using the scanning Fabry-Perot (FP) interferometer PUMA (Rosado et al. 1995). The velocity fields as well as the rotation curves (RCs) along the major axis of both galaxies in the pair were obtained. From those we determined that the mass-ratio between the two galaxies has a value within $1 / 63$ to $1 / 168$.

The RC of the host galaxy, NGC 3893, allows us to study the mass distribution of the different galaxy components (stellar disk and bulge, gas and dark matter halo). Fig. 1 (left) shows the measured RC of NGC 3893 together with the contributions of its different components (disk and dark matter halo) used as boundary conditions in the numerical simulations. It is necessary to recall that the fit is not unique first of all because the mass-to-light ratio of the disk $(\mathrm{M} / \mathrm{L})_{D I S K}$ is not well known (it would be necessary to fit population synthesis models to the disk photometry and/or spectroscopy in order to better determine this parameter) and because one can fit different DM halo density distributions. Thus, the $(\mathrm{M} / \mathrm{L})_{D I S K}$ as well as the dark matter (DM) halo properties -central density $\rho_{0}$ and core radius $r_{0}$, are free parameters. The DM halo can be either a pseudo-isothermal sphere (Begeman 1987), a NFW profile (Navarro et al. 1996), or a Hernquist (1990) profile.

\section{Numerical simulations}

\subsection{Preliminary (without gas) simulations}

As a first step, we conducted numerical simulations using GADGET2 and the geometry displayed in Fig. 1 (right) as discussed in Rosado et al. (2008). These preliminary simulations were done considering the satellite galaxy, NGC 3896 as a point-mass in a retrograde elliptic orbit $(\mathrm{e}=0.79)$. A particularity of these simulations is the fact that we did not consider the presence of gas. Thus, the N-body simulations consider NGC 3893 as a galaxy formed of a stellar disk and a dark matter halo with 10000 and 60000 particles, respectively. The main result of these simulations was that in order to develop a grand-design spiral structure the mass-ratio between satellite and host galaxies could not
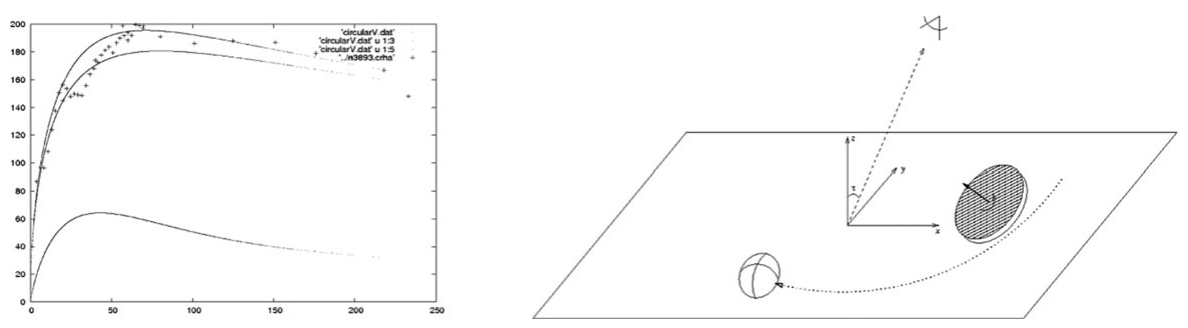

Figure 1. (left) Rotation Curve of the host galaxy NGC 3893 obtained from PUMA observations (crosses for $\mathrm{R} \leqslant 80$ arcsec) and $\mathrm{HI}$ observations (crosses for $\mathrm{R} \geqslant 80$ arcsec, from Verheijen \& Sancisi 2001). The $\mathrm{X}$ axis corresponds to galactocentric radius in units of arcsec and the $\mathrm{Y}$ axis to the rotation velocity in $\mathrm{km} \mathrm{s}^{-1}$. The solid lines correspond to (from bottom to top) the contributions of a stellar plus gaseous disk, of a DM halo and to the sum of disk and halo components. (right) Geometry of the encounter in KPG 302 as deduced from our kinematics. 
Table 1. Typical Parameters of the Model of the Host NGC 3893.

\begin{tabular}{|l|ccc|cc|c|c|c|c|}
\hline $\begin{array}{l}\text { Galaxy } \\
\text { Component }\end{array}$ & $\left.\begin{array}{c}\text { Mass } \\
{\left[\mathbf{2 . 2} \times \mathbf{1 0}^{11}\right.}\end{array} \mathbf{M} \odot\right]$ & $\mathbf{r}_{0}[\mathbf{k p c}]$ & $\mathbf{R}_{\text {cut }}[\mathbf{k p c}]$ & $\mathbf{Z}_{0}[\mathbf{p c}]$ & $\begin{array}{c}\text { Number of } \\
\text { Particles }\end{array}$ \\
\hline DM Halo & 1.004 & $\mid$ & 7.5 & 160 & - & 360448 \\
\hline Stellar Disk & 0.039 & $\mid$ & 1.8 & 12 & 280 & 58982 \\
\hline Gas & 0.0043 & $\mid$ & 1.8 & 12 & 30 & 6554 \\
\hline
\end{tabular}

be too low: $\mathrm{M}_{2} / \mathrm{M}_{1}$ should be of about $1 / 10$. However, the pair of galaxies merged too fast (within $500 \mathrm{Myr}$ ).

\subsection{More realistic simulations}

In a new series of simulations carried out with GADGET2 code we consider the same components for the host galaxy (stellar disk and dark matter halo) but the satellite galaxy is no longer considered as a point-mass. Also, we have included gas in the disk component to run the simulations.

\subsubsection{Galaxy models}

We use a model of galaxy similar to the one described in Barnes \& Hernquist (1996). The model considers three types of matter: stars, dark matter, and interstellar gas. Both stars and DM are governed by the collisionless Boltzmann equation while the gas is modeled by the laws of a compressible fluid, with the addition of gravitational forces. A hierarchical N-body code simulates the collisionless components and smoothed particle hydrodynamics (SPH) evolves the gas. Consequently, all the components are represented by particles. The stellar and gas disks have exponential density profiles (Freeman 1970). The gas is considered isothermal at $\mathrm{T}=10^{4} \mathrm{~K}$ and having the vertical scale height $\mathrm{Z}_{0} \sim 10 \%$ of the stellar disk. An artificial viscosity in the equations of motion is included to handle the shocks. The gas amounts to $10 \%$ of the stellar disk mass. The DM halo density profile is adopted either from Hernquist (1990), from Begeman (1987) (for a pseudo-isothermal sphere) or from Navarro et al. (1996) (NFW density profile). With these assumptions, we fit to the rotation curve of the host galaxy (NGC 3893) obtained from our kinematical observations, a component representing the disk (stars and gas) contribution and a DM halo (see for example Fig. 1). Table 1 lists typical values for the host galaxy with NFW halo.

The satellite galaxy (in this case NGC 3896) is modeled as a Plummer sphere. Typical mass-ratios between galaxies are $1 / 16$ to $1 / 10$ (see Sect. 3.1 ).
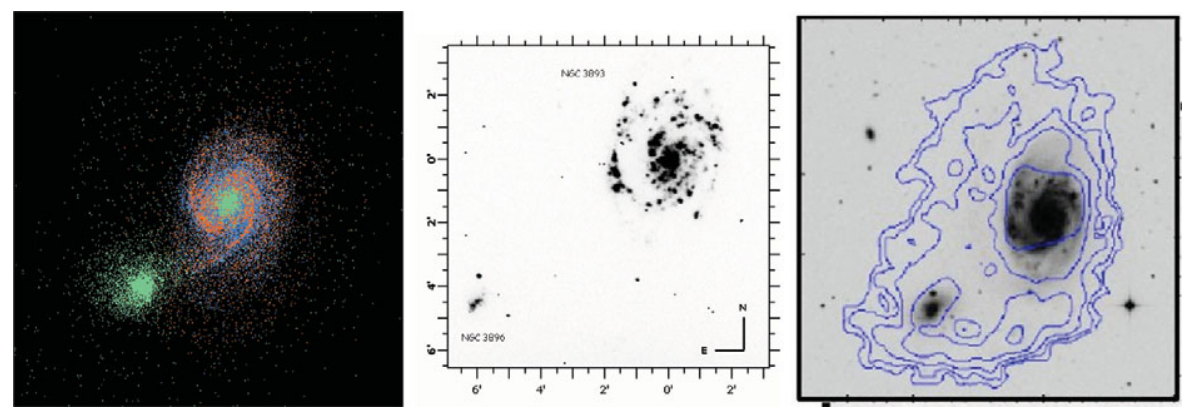

Figure 2. (left) Snapshot of one of our simulations with multiple passages after 850 Myrs. (center) PUMA direct $\mathrm{H} \alpha$ image of KPG 302. (right) HI contours overlayed in a continuum image of KPG 302. 


\subsubsection{Assumptions on the orbital parameters and results}

We have considered only parabolic orbits which seem to be the more frequent according to Benson (2005). The modeling of the galaxy interaction is started by placing the two model galaxies (host and satellite) in the computed orbits of the solutions of the 2-body problem for two mass points, for $\mathrm{R} \geqslant 4 \mathrm{R}_{\text {cut }}$. We have considered two main cases: one interaction and two or multiple passages caused by dynamical friction.

Fig. 2 shows the result of one of the numerical simulations considering parameters similar to the ones listed in Table 1, a direct parabolic interaction with multiple passages after 850 Myrs. The resemblance with the modeled pair KPG 302 is encouraging. The measured velocity map has been also confronted with the predictions of the simulations at that epoch and the agreement was very good. Following the evolution of the system in the simulation we see that after the second passage the pair merges at about 1700 Myrs (the simulation lasted 2500 Myrs) which is longer than the 500 Myrs merging time of the simulations with the satellite represented as a point mass. This indicates that M51-type pairs can have several passages lasting very long times. Also, it is seen that the satellite galaxy looses stars and gas in every passage. This could explain why the mass-ratio deduced from the kinematical observations $(1 / 63$ to $1 / 168)$ is considerably smaller than the mass-ratios used in the simulations that could trigger spiral structure in the host galaxy $(1 / 10$ to $1 / 16)$.

The observational data are so rich that a very extensive comparison can help us to gain insights about the parameters we have selected and to constrain them more. For example, the pitch angle of the spiral pattern of NGC 3893 measured by Kranz et al. (2003) from NIR observations is of 19 degrees while the numerical simulations predict pitch angles that depend on the density distribution of the DM halo being better suited those with pseudo-isothermal density profiles. We are still conducting comprehensive sets of simulations in order to take into account the different observations on this galaxy pair.

In conclusion, numerical simulations directly confronted with observations (on the morphology and kinematics) of M-51 galaxy pairs are important in the study of the evolution of these systems and the formation of grand-design spiral patterns among other interesting issues. Our simulations show that in order to produce a grand-design spiral pattern it is required: a gaseous component, a direct collision and multiple passages (at least for KPG 302). There is some indication that more open spiral patterns are more easily produced by pseudo-isothermal DM halos. Still it is important to include a bulge in the numerical simulations. Although the mass considered in a bulge component is small, its presence could help in the stability of the system against bar formation.

\section{References}

Barnes, J. E. \& Hernquist, L. 1996, ApJ, 471, 115

Begeman, K. G. 1987, PhD Thesis, Groenigen University

Benson, A. J. 2005, MNRAS, 358, 551

Freeman, K. C. 1970, ApJ, 160, 811

Fuentes-Carrera, I., Rosado, M., Amram, P., Salo, H., \& Laurikainen, E. I. 2007, A\& A, 415, 451 Hernquist, L. 1990, ApJ, 356, 359

Karachentsev, I. D. 1972, Cat. of isol. pairs of gal. in the northern hem., Soobshch.Spets.Astrofiz.Obs, 7, 1

Kranz, T., Slyz, A., \& Rix, H.-W. 2003, ApJ, 586, 143

Navarro,J. F., Frenk,C. S. \& White,S. D. M.. 1996, ApJ, 462, 563

Rosado, M., Langarica, R., \& Bernal, A., et al. 1995, RMxAC , 3, 263

Rosado, M., Fuentes-Carrera, I., \& Gabbasov, R., et al. 2008, RMxAC, 32, 147

Verheijen, M. A. W.. \& Sancisi, R. 2001, A\&A, 370, 765 